\title{
Dealing with Disaster
}

T

The accident and subsequent oil leak from the deepdrilling rig in the Gulf of Mexico presents us with some stark choices. As restorationists, we like to gather information, make plans based on sound science, source appropriate materials, begin the physical and biological aspects of project implementation, then monitor and carry out adaptive management.

But what if there is no time to gather information, run predictive models, find sufficient resources, and implement procedures at a comfortable pace? At the time of writing, oil is still leaking into the Gulf and being thrown up onto shores and wetlands - and urgent action is being taken. So, knowing that we don't have the time we would like to take, should we wait until the immediate work of preventing further leakage is complete, until the booms and berms are all in place, then deal with the aftermath? Or should we be pitching in now to provide advice and action to mitigate the worst effects and plan for the time when we can restore the system to a functioning state and, beyond that, restore the system to its fullest potential? I suspect that what will emerge will be a combination of approaches, dependent on local conditions and resources (natural, social, and economic capital). Let us endeavor to do our best in extremely difficult circumstances.

This issue is devoted to restoration in Mexico, which makes the timing poignant in the light of the spill. There are wonderful examples of restoration throughout Mexico highlighted in the following pages from large-scale government-backed and delivered programs to locally based actions, in a land rich in biodiversity and cultural history. It is particularly heartening to see the fruits of collaboration between local communities, colleges, and universities, melding indigenous knowledge with sound science. I congratulate the contributors for their hard work and dedication to reconnecting nature with culture.

\author{
Jim Harris \\ Chair \\ Society for Ecological Restoration International
}

Ecological Restoration Vol. 28, No. 3, 2010

ISSN 1522-4740 E-ISSN 1543-4079

(C2010 by the Board of Regents of the University of Wisconsin System. 